\title{
Flash-Free Orthodontic Adhesive System Bonded on CAD/CAM Produced Resin Restorations
}

\author{
DENISA MARIA GOLBAN ${ }^{1}$, RADU NEGRU², CAMELIA SZUHANEK, MEDA LAVINIA NEGRUTIU ${ }^{1}$, LIVIU MARSAVINA², \\ VIRGIL FLORIN DUMA ${ }^{2,3}$, COSMIN SINESCU ${ }^{1}$, LILIANA POROJAN ${ }^{1}$ \\ ${ }^{1}$ Victor Babes University of Medicine and Pharmacy of Timisoara, Faculty of Dental Medicine, 2 Eftimie Murgu Sq., 300041 \\ Timisoara, Romania \\ 2Politehnica University of Timisoara, Faculty of Mechanical Engineering, 1 Mihai Viteazu Ave., 300006 Timisoara, Romania \\ ${ }^{3}$ Aurel Vlaicu University of Arad, 30M Optomechatronics Group, Faculty of Engineering, 77 Revolutiei Ave., 310130 Arad, \\ Romania
}

\begin{abstract}
Flash-Free Orthodontic Adhesive System represents the latest technology in the field of orthodontics. In the present study the bonding technique is applied on CAD/CAM produced resin crowns. Ten resin blocks were shaped after an upper premolar crown design from the 3Shape Library, and they were divided ino two groups: on Group 1, control, Clarity Advanced braces (3M Unitek, Monrovia, CA, USA) were bonded using XT Adhesive Paste (3M Unitek, Monrovia, CA, USA), while on Groups 2 same aesthetic brackets were applied, using the APC Flash-Free Adhesive Coated Appliance System (3M Unitek, Monrovia, CA, USA). Bracket positioning time was recorded for each sample. Zwick Zoel Z005 testing machine was used to measure the shear bond strength (SBS) at which debonding takes place. Adhesive remnant index (ARI) was registered, as well as presence or absence of adhesive flash and accuracy of flash cleaning before curing. Group 1 showed a mean bonding time of $46.4 \mathrm{~s}$, while Group 2 needed about $20.6 \mathrm{~s}$ for each bracket bonding. Mean SBS values for both groups are considered clinically acceptable (6.27 MPa for Group 1 and 7.77 MPa for Group 2). ARI scores were zero for all samples and after debonding, on group 1 can be observed the adhesive flash. In conclusion, APC Flash-Free Coated Appliance System showed reduced time for bracket positioning compared to the conventional technique. APC Flash-Free System showed acceptable SBS values, higher than the uncoated brackets. No composite was left on the resin crown after debonding, the adhesive flash resulted in the conventional technique remaining on the edges of the debonded brackets
\end{abstract}

Keywords: APC Flash-Free, CAD/CAM produced resin restoration, bonding time, shear bond strength (SBS), adhesive remnant index.

It is well known that modern day orthodontic treatment is applied to a large spectrum of patients, of all ages and all anomalies. When an interdisciplinary collaboration between orthodontics and prosthodontics is needed, the patient will receive temporary crowns until the fixed appliances are removed [1].

Computer aided design/computer aided machine (CAD/ CAM) was introduced in dentistry in 1980, having, compared to conventional crown making techniques, the advantages of shorter working time, lower costs, higher restoration homogeneity and reproducibility, all with an acceptable marginal adaptation [2,3]. The progress made in the field of resin-based materials gave technicians the possibility of using this technique to produce temporary crowns strong enough to resist for an average orthodontic treatment. Resin, compared to ceramic, can be easily adjusted or repaired [4].

The main disadvantage of CAD/CAM produced resin restorations results, ironically, from the greater strength of the material. Due to the high degree of polymerisation, it is hard to achieve a high bond strength between the resin crown and adhesive cements [5]. This is the reason why bracket bonding to these surfaces can be difficult. Orthodontic products biocompatibility is very important in achieving clinical results $[15,16]$.

Several authors [6-10] studied the bond strength of different adhesives used to attach metal brackets to resin surfaces, but there is no research on the bond between a ceramic bracket and a resin restoration. 3M Unitek
(Monrovia, CA, USA) offers this new concept, APC FlashFree Adhesive Coated Appliance System, which they support presents higher bond strength than the same brackets, Clarity Advanced (3M Unitek, Monrovia, CA, USA), bonded by the classical method, where adhesive is applied by the clinician on the bracket base [11].

In the present paper, we studied the shear bond strength formed between APC Flash-Free aesthetic brackets and a CAD/CAM produced resin teeth without any surface treatment prior bonding.

\section{Experimental part \\ Material and methods}

An upper premolar crown shape was selected from the Library provided by 3Shape Dental System, software for 3 D reconstruction, on which a rectangular base was digitally added. Ten poly methyl methacrylate (PMMA) blocks were identically shaped with the help of the CAD/ CAM technology. No type of surface treatment was applied besides rinsing, drying and Transbond XT Light Cure Adhesive Primer (3M Unitek, Monrovia, CA, USA), cured for 10 seconds.

All brackets were positioned by the same clinician and all individual times were recorded. On half of the premolar shaped resin blocks (Group 1- control), Clarity Advanced braces (3M Unitek, Monrovia, CA, USA) were bonded using a classic bonding technique, using Transbond XT Adhesive Paste (3M Unitek, Monrovia, CA, USA) applied by the clinician, in office, on the bracket base. The adhesive

*email: cameliaszuhanek@umftro; Phone: 0040724251240 
excess was removed during bracket positioning. APC FlashFree Adhesive Coated Appliance System was applied on the other half resin blocks (Group 2). Curing light was applied on each bracket from all directions for a total of 20 S.

Zwick Zoel Z005 testing machine was used to measure the shear bond strength (SBS) on which debonding takes place. The samples were mounted in the jug of the machine and the force was applied on the bracket with the help of a metal chisel, at a speed of $0.5 \mathrm{~mm} / \mathrm{s}$, parallel with the fake tooth axis, in a oclussal-gingival direction (fig. 1).

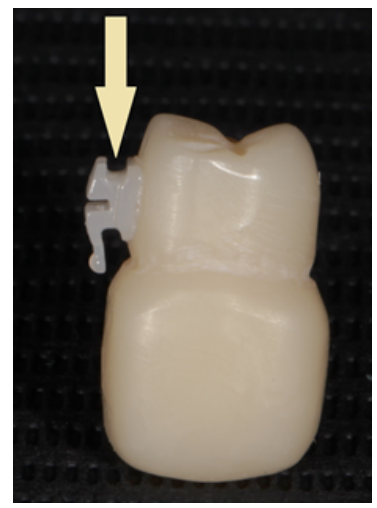

Fig. 1. Occlusal-gingival direction of the force applied for debonding.

All bracket bases were photographed after debonding using Canon 60D camera. Adhesive remnant index was established as following: [12]

$0=$ the entire adhesive is left on the bracket base;

$1=$ more than half of the adhesive is left on the bracket base;

$2=$ less than half of the adhesive is left on the bracket base;

$3=$ no adhesive is left on the bracket base.

On the digital photographs was also possible to determine the existence of adhesive flash and the accuracy of flash cleaning before curing.

\section{Results and discussions}

The clinician achieved shorter time for APC Flash Free brackets positioning (table 1). The mean time needed to place the adhesive paste on the uncoated brackets and remove flash adhesive took more time, even if the crowns were identical (table 2).

\begin{tabular}{|c|c|c}
\hline Method & Time [s] & \\
\hline Conventional Direct Bonding & 49 & Table 1 \\
& 43 & THE AMOUNT \\
& 46 & OF TIME \\
& 45 & NEEDED IN \\
& 49 & ORDER TO \\
& 23 & BOND EACH \\
& 19 & BRACKET. \\
& 20 & \\
& 20 & \\
& 21 \\
& &
\end{tabular}

Table 2

MEAN BRACKET BONDING TIME FOR THE TWO GROUPS

\begin{tabular}{|c|c|c|}
\hline Method & $\begin{array}{c}\text { Mean bracket } \\
\text { bonding time [s] }\end{array}$ & $\begin{array}{c}\text { Standard } \\
\text { deviation [s] } \\
\text { Conventional Direct } \\
\begin{array}{c}\text { Bonding } \\
\text { APC Flash Free }\end{array}\end{array}$ \\
\begin{tabular}{c} 
Adhesive System \\
\hline
\end{tabular} & 20.6 & 2.60 \\
\hline
\end{tabular}

The SBS values obtained after $24 \mathrm{~h}$ for APC Flash Free brackets is higher than the Clarity Advanced brackets bonded through conventional methods (table 4). One of

\begin{tabular}{|c|c|c|}
\hline Type of & SBS [MPa] & \\
\hline brackets & & \\
& 7.73 & \\
& 8.52 & Table 3 \\
& 4.61 & SHEAR BOND STRENGTH \\
(SBS) [MPa]
\end{tabular}

the samples was left out of the study, the force that debonded the bracket being very small, considering itwas a tainted resin surface.

Adhesive remnant index values are 0 for all samples showing the low adherence on the resin tooth surface when no preatreatment is applied (table 5). Considering that the entire adhesive was separated from the resin crowns, on the images obtained after debonding, it can be observed the flash of adhesive present in Group 1, of uncoated brackets(fig.2, 3).

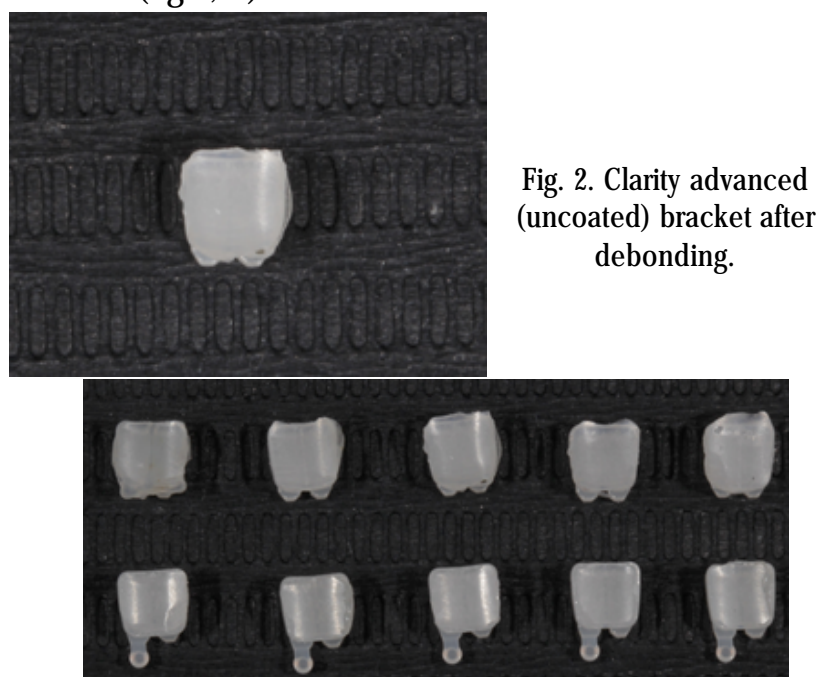

Fig. 3. Brackets after debonding: Upper row-Group 1, Lower rowGroup 2

APC Flash-Free brackets are individually packed and for bonding it only needs correct positioning and pressing on the tooth surface, saving valuable time. The time obtained in this study was approximately $20 \mathrm{~s}$, half the time needed for bonding brackets by the traditional technique. Similar results were found in other studies with a time difference of $10 \mathrm{~s}$ [13] to $20 \mathrm{~s}$ [14] between groups. Adhesive excess that needs to be cleaned before curing disturbs the position of the bracket, implying more time for a correct placing.

Mean shear bond strength values found in both groups is considered clinically accepted (6.27 MPa for Group 1 and $7.77 \mathrm{MPa}$ for Group 2). Without any type of resin surface 
treatment to increase the roughness of the crown, Brunharo et al [6] obtained SBS of $2.30 \mathrm{MPa}$, but manage to increase the values as high as $12.02 \mathrm{MPa}$ when using bicarbonate sandblasting prior ro bonding, even higher than the results obtained after the use of bur (approximately $9.26 \mathrm{MPa}$ ). Goymen et al [7] did not manage to increase the bond strength with any of the pretreatments chosen (acid-etch, sandblasting, laser irradiation).

Al Jabbari et al [8] came to the conclusion that bond strength is influenced by more factors that just surface treatment, including the resin from which the crown if made or local factors that produce material ageing.

The smoothness of the resin surface before bracket bonding is translated through the ARI index equal to zero for all samples.

Even with the effortfor a proper flash removal, a certain amount of adhesive can be observed retained on the bracket base after debonding (fig. 2, 3). On the lower row presented on figure 3, APC Flash Free brackets present minimum to none adhesive on the base edge, theoretically with no risk of adhesive colouring.

\section{Conclusions}

Bonding with APC Flash Free System takes less than half compared to conventional direct bonding of uncoated brackets. In the attempt of an accurate clean, more time for bracket adjustment is needed.

APC Flash Free System, even if itshow ed low SBS values, they are clinically acceptable if the crown surface is correctly washed and dried.

ARI scores for all samples was zero, meaning all the adhesive was left on the bracket base after bonding.

APC Flash Brackets indeed showed no flash adhesive, while for the convetional bonded group, the flash is visible with the naked eye and on the digital images.

Acknowledgements: This research was supported by the Romanian National Authority for Scientific Research, CNDI-UEFISCDI ProjectPN-IIIP2-2.1-PTE-2016-0181 (http://3om-group-optomechatronics.ro/). Cosmin Sinescu also acknowledges the support of the Victor Babes University of medicine and Pharmacy of Timisoara (Grant PIII-C2-PCFI (2015-2016), DENTALOCT).

\section{References}

1. M. CURMELATO, E.M. LIMA, L.B. OSORIO, E.G. MOTA, L.M. MENEZES, S.M.D. RIZZATTO, Effect of surface treatment of prefabricated teeth on shear bond strength of orthodontic brackets, Dental Press J Orthod 2017, 22(4), 47-52.

2. S. LAUVAHUTANON, H. TAKAHASHI, M. SHIOZAWA, N. IWASAKI, Y. ASAKAWA, M. OKI, W.J. FINGER, Mechanical properties of composite resin blocks for CAD/CAM, Dental Mat J 2014, 33(5), 705-710.
3. L. SANDU, F. TOPALA, S. POROJ AN, Marginal design evaluation for CAM obtained Zirconia based crown frameworks, Adv Mat Res 2011, 213, 349-354.

4. L. POROJAN, F. TOPALA, Failure analisys of various monolithic posterior aesthetic dental crowns using finite element method, J Phys: Conf Ser 2017, 885.

5. A. KAWAGUCHI-UEMURA, A. MINE, M. MATSUMOTO, Y. TAJIRI, M. HIGASHI, T. KABETANI, R. HAGINO, D. IMAI, T. MINAMINO, I. MIURA, H. YATANI, Adhesion procedure for CAD/CAM resin crown bonding: Reduction of bond strengths due to artificial saliva contamination, J Prosth Res 2018, 62, 177-183.

6. I.H.V.P. BRUNHARO, D.J. FERNANDES, M.S. MIRANDA, F. ARTESE, Influence of surface treatment on shear bond strength of orthodontic brackets, Dental Press J Orthod 2013 May-J une, 18(3), 54-62.

7. M. GOYMEN, T. TOPCOGLU, S. TOPCUOGLU, H. AKIN, Effect of temporary crown materials and surface roughening methods on shear bond strengths of orthodontic brackets, Photomed Laser Surg 2015, 33(2).

8. Y.S. AL JABBARI, S.M. AL TAWEEL, M. AL RIFAIY, M.Q. ALQAHTANI, T. KOUTSOUKIS, S. ZINELOS, Effects of surface treatment and artificial aging on the shear bond strength of orthodontic brackets bonded to four different provisional restorations, Angle Orthod 2014 Jul, 84(4), 649-55.

9. H.I. SOON, D.S. GILL, S.P. JONES, A study to investigate the bond strength of orthodontic brackets bonded to prosthetic acrylic teeth, J Orthod 2015 Sep, 42(3), 192-199.

10. A.R.W.S. WAN, M. SHERRIFF, D. BISTER, J. SEEHRA, Bond strength of stainless steel orthodontic brackets bonded to prefabricated acrylic teeth, J Orthod 2017 J un, 44(2), 105-109.

11. M. FOERSCH, C. SCHUSTER, R. K. RAHIMI, H. WEHREBEIN, C. JACOBS, A new flash-free orthodontic adhesive system: A first clinical and stereomoscopic study, Angle Orthod 2016, 86, 260-264.

12. J. ARTUN, S. BERGLAND, Clinical trials with crystal growth conditioning as an alternative to acid-etch enamel pretreatment. Am J Orthod Dentofacial Orthop 1984, 85, 333-340.

13. M. LEE, G. KANAVAKIS, Comparison of shear bond strength and bonding time of a novel flash-free bonding system, Angle Orthod 2016, 86, 265-270.

14. M. FOERSCH, C. SCHUSTER, R. K. RAHIMI, H. WEHREBEIN, C. JACOBS, A new flash-free orthodontic adhesive system: A first clinical and stereomoscopic study, Angle Orthod 2016, 86, 260-264.

15. SZUHANEK, C., JIANU, R.,SCHILLER,E., GRIGORE, A., LEVAI, C., POPA, A., - Acrylic versus Silicone in Interceptive Orthodontics. Mat. Plast., 53, no. 4, 2016 p. 759-760.

16. SZUHANEK, C., JIANU, R., CIRCIUMARU, L., NEGRUTIU, M., SINESCU, C., CLONDA,C.S., SCHILLER, E., POPA, A., GRIGORE, A., Microstructural Changes in Orthodontic Archwires after Alternative Bending Techniques. Rev.Chim.(Bucharest), 67, no.11, 2016, p.23632364.

Manuscript received: 2.08 .2108 